\title{
CHARACTERIZATION OF REGULAR HAUSDORFF MOMENT SEQUENCES
}

\section{J. S. MAC NERNEY}

The theorem of this paper is suggested to the author by H. S. Shapiro's proposal [3], to the effect that

$$
\operatorname{Lim}_{n \rightarrow \infty} \sum_{k=0}^{n}(-1)^{k} f(k / n)\left(\begin{array}{l}
n \\
k
\end{array}\right) / 2^{n}=0
$$

for each $f$ in $C[0,1]$, the class of continuous functions from $[0,1]$ to the complex numbers. Indeed, we find that this proposal is precisely the assertion that the sequence $\mu, \mu_{n}=1 / 2^{n}(n=0,1, \cdots)$, is a regular Hausdorff moment sequence with limit 0 . The latter assertion is well known: for relevant ideas, one may consult [4, Chapters 14 and 16] and, especially, $[4$, p. 309$]$ regarding the Euler-Knopp mean $(E, 1 / 2)$ associated with the aforementioned sequence $\mu$. The connecting link is the following result.

THEOREM. In order that the infinite complex number sequence $\mu$, with $\mu_{0}=1$, be a regular Hausdorff moment sequence, it is necessary and suffcient that, for each $f$ in $C[0,1]$, the limit

$$
L(f)=\operatorname{Lim}_{n \rightarrow \infty} \sum_{k=0}^{n}(-1)^{n-k} f(k / n)\left(\begin{array}{l}
n \\
k
\end{array}\right) \Delta^{n-k} \mu_{k}
$$

exist, and in this case, for each $f$ in $C[0,1], L(f)=f(1) \cdot \operatorname{Lim}_{n \rightarrow \infty} \mu_{n}$.

INDICATION OF PROOF. If $L(f)$ exists for each $f$ in $C[0,1]$, then there is a number $B$ such that

$$
\sum_{k=0}^{n}\left(\begin{array}{l}
n \\
k
\end{array}\right)\left|\Delta^{n-k} \mu_{k}\right| \leqq B \quad(n=0,1, \cdots) .
$$

This is an application of the "principle of uniform boundedness" in the linear space $C[0,1]$, normed as usual by $\|f\|=$ L.U.B. $|f(x)|$ : see [1, Chapter 2], especially the remarks on pp. 80-81 thereof. By a theorem due to F. Riesz [4, p. 271], (3) is equivalent to the existence of a complex-valued function $\phi$, of bounded variation on $[0,1]$, such that

Presented to the Society, November 15, 1963; received by the editors February $27,1963$. 


$$
\phi(0)=0 \quad \text { and } \quad \mu_{n}=\int_{0}^{1} I^{n} d \phi \quad(n=0,1, \cdots),
$$

where $I$ denotes the identity function on $[0,1]$. In this circumstance, the sequence $\mu$ has the limit $\phi(1)-\phi(1-)$, and there is a theorem due to F. Hausdorff $[4$, p. 309] to the effect that $\mu$ is regular only in case $\phi$ is continuous at 0 , i.e., if and only if $\phi(0+)=0$.

From the preceding considerations, together with the Weierstrass theorem on uniform approximation by polynomials, we see that the theorem will be established once we have the following lemma.

Lemмa. If $\mu$ is the Hausdorff moment sequence given by (4) with $\phi$ of bounded variation then, for each polynomial $g$, the sequence $\lambda(g)$,

$$
\lambda_{n}(g)=\sum_{k=0}^{n}\left\{(-1)^{n-k} g(k / n)\left(\begin{array}{l}
n \\
k
\end{array}\right) \Delta^{n-k} \mu_{k}\right\}-(-1)^{n} g(0) \phi(0+),
$$

converges and has the limit $g(1)[\phi(1)-\phi(1-)]$.

Proof of Lemma. Starting with a positive integer $n$, and the binomial theorem in the form

$$
h_{0}(z)=\sum_{k=0}^{n} z^{k}\left(\begin{array}{l}
n \\
k
\end{array}\right)(1-I)^{n-k} I^{k}=(1-I+z I)^{n},
$$

one establishes inductively the fact that if $p$ is a positive integer not greater than $n$ then $h_{p}(z)=z h_{p-1}^{\prime}(z)$ is given by

$$
\sum_{k=0}^{n} z^{k} k^{p}\left(\begin{array}{l}
n \\
k
\end{array}\right)(1-I)^{n-k} I^{k}=\sum_{q=1}^{p}\left(\begin{array}{l}
n \\
q
\end{array}\right) d_{p, q}(1-I+z I)^{n-q}(z I)^{q},
$$

where $d_{p, 1}=1, d_{p, p}=p$ !, and $d_{p+1, q}=\left(d_{p, q-1}+d_{p, q}\right) q$ if $1<q<p$ : see $\left[2\right.$, p. 57] for a similar use of the numbers $d_{p, q}$. Now by taking $z$ to be -1 in (7), and multiplying by $(-1)^{n}$, we see that

$$
\sum_{k=0}^{n}(-1)^{n-k} k^{p}\left(\begin{array}{l}
n \\
k
\end{array}\right)(1-I)^{n-k} I^{k}=\sum_{q=1}^{p}\left(\begin{array}{l}
n \\
q
\end{array}\right) d_{p, q}(2 I-1)^{n-q} I^{q} .
$$

From (6), we see that for each positive integer $n$

$$
\lambda_{n}\left(I^{0}\right)=\int_{0+}^{1-}(2 I-1)^{n} d \phi+\phi(1)-\phi(1-),
$$

which clearly has limit $\phi(1)-\phi(1-)$ as $n \rightarrow \infty$. On the other hand. we see from (8) that, for $0<p<n$, 


$$
\lambda_{n}\left(I^{p}\right)=\sum_{q=1}^{p}(1 / n)^{p}\left(\begin{array}{l}
n \\
q
\end{array}\right) d_{p, q} \int_{0}^{1}(2 I-1)^{n-q} I^{q} d \phi,
$$

which also has limit $\phi(1)-\phi(1-)$ as $n \rightarrow \infty$. This completes the proof.

The theorem, now established, seems a natural companion to the following proposition, which is easily established by application of the "principle of uniform boundedness" and computation with Bernstein polynomials, and which we state without proof:

Proposition. In order that the infinite complex number sequence $\mu$ be a Hausdorff moment sequence, it is necessary and sufficient that, for each $f$ in $C[0,1]$, the limit

$$
M(f)=\operatorname{Lim}_{n \rightarrow \infty} \sum_{k=0}^{n} f(k / n)\left(\begin{array}{l}
n \\
k
\end{array}\right) \Delta^{n-k} \mu_{k}
$$

exist, and in this case, for each $f$ in $C[0,1], M(f)=\int_{0}^{1} f d \phi$ where $\phi$ is a function of bounded variation having $\mu$ as its moment sequence.

\section{BIBLIOGRAPHY}

1. N. Dunford and J. T. Schwartz, Linear operators, Interscience, New York, 1958.

2. J. S. Mac Nerney, Hermitian moment sequences, Trans. Amer. Math. Soc. 103 (1962), 45-81.

3. H. S. Shapiro, Proposal of advanced problem 5060, Amer. Math. Monthly 69 (1962), 1013.

4. H. S. Wall, Analytic theory of continued fractions, Van Nostrand, New York, 1948.

University of North Carolina at Chapel Hill 